\title{
Room Temperature Carrier Kinetics in the W-type GaInAsSb/InAs/AlSb Quantum Well Structure Emitting in Mid-Infrared Spectral Range
}

\author{
M. Syperek ${ }^{a, *}$, K. Ryczko ${ }^{a}$, M. DAllner $^{b}$, M. Dyksik $^{a}$, M. Motyka $^{a}$, M. KAmp $^{b}$, \\ S. HÖFLING ${ }^{b, c}$, J. MisiewiCZ ${ }^{a}$ AND G. SĘK \\ ${ }^{a}$ Laboratory for Optical Spectroscopy of Nanostructures, Department of Experimental Physics, \\ Faculty of Fundamental Problems of Technology, Wrocław University of Science and Technology, \\ Wybrzeże S. Wyspiańskiego 27, 50-370 Wrocław, Poland \\ ${ }^{b}$ Technische Physik, University of Würzburg and Wilhelm-Conrad-Röntgen-Research Center \\ for Complex Material Systems, Am Hubland, D-97074 Würzburg, Germany \\ ${ }^{c}$ School of Physics and Astronomy, University of St. Andrews, North Haugh, \\ KY16 9SS St. Andrews, United Kingdom
}

\begin{abstract}
Room temperature carrier kinetics has been investigated in the type-II W-design AlSb/InAs/ $\mathrm{Ga}_{0.80} \mathrm{In}_{0.20} \mathrm{As}_{0.15} \mathrm{Sb}_{0.85} / \mathrm{InAs} / \mathrm{AlSb}$ quantum well emitting in the mid-infrared spectral range (at $2.54 \mu \mathrm{m}$ ). A timeresolved reflectance technique, employing the non-degenerated pump-probe scheme, has been used as a main experimental tool. Based on that, a primary carrier relaxation time of $2.3 \pm 0.2$ ps has been found, and attributed to the initial carrier cooling process within the quantum well states, while going towards the ground state via the carrier-optical phonon scattering mechanism. The decay of a quasi-equilibrium carrier population at the quantum well ground states is primarily governed by two relaxation channels: (i) radiative recombination within distribution of spatially separated electrons and holes that occurs in the nanosecond time scale, and (ii) the hole tunnelling out of its confining potential, characterized by a $240 \pm 10$ ps time constant
\end{abstract}

DOI: 10.12693/APhysPolA.130.1224

PACS/topics: 73.21.Fg, 78.47.D-, 78.30.Fs, 78.47.jg, 78.55.Cr, 78.67.De

\section{Introduction}

The type-II W-design quantum wells (QWs) of a staggered band alignment, based on an InAs/GaInSb combination, have been developed as a key element of the gain medium of interband cascade lasers (ICLs) emitting in the mid-infrared spectral range $(2.5-6 \mu \mathrm{m})$ [1]. ICLs have been proven to have a huge application potential in optical gas sensing of medically and environmentally relevant gases, and to surpass the systems based on their counterparts, e.g. quantum cascade lasers, in some performances. In spite of that, some of the properties of the active region in this experimentally demanding spectral range are still not well known, and this concerns also carrier dynamics, on which only very initial works have been reported in the 90's [2-6].

Several types of experimental methods have been used in order to study carrier dynamics in QW systems of that kind, including: time-resolved differential transmission [2, 5], time-resolved photoluminescence up-conversion $[3,5,6]$, or photoconductive response technique [4]. All of them employ highly intensive laser pulses in order to track carrier relaxation processes in multiple QWs of the considered

\footnotetext{
*corresponding author; e-mail: marcin.syperek@pwr.edu.pl
}

type. In this work, we use an experimentally challenging time-resolved reflectivity measurement at room temperature in the mid-infrared spectral range, based on the non-degenerated pump-probe technique utilizing weak pJ pulses. This type of experiment allows monitoring of the initial carrier relaxation process at low carrier densities photoinjected into the W-type $\mathrm{AlSb} / \mathrm{InAs} / \mathrm{Ga}_{0.80} \mathrm{In}_{0.20} \mathrm{As}_{0.15} \mathrm{Sb}_{0.85} / \mathrm{InAs} / \mathrm{AlSb} \mathrm{QW}$ as well as subsequent relaxation of a quasi-equilibrium carrier population at the QW ground states (GS) by radiative and nonradiative recombination processes. Since it is based on the reflectance of the probe pulse, this experimental approach does not require any special treatment of the sample, in particular such that allow the probe laser beam to be transmitted through the entire structure. Moreover, the use of weak excitation prevents unwanted effects related to the presence of the Auger type scattering, which most likely dominates carrier relaxation scenarios at intensive excitation. The great advantage of the pump-probe experiment over the photoluminescence one is that it is able to separately test changes in an electron and a hole distribution functions, in contrast to the emission-type experiments, where only the joint electron-hole population is examined over time.

In other to strengthen the discussion concerning results of the time-resolved experiment, theoretical calculation of the QW band structure has been performed within the framework of an 8-band $\boldsymbol{k} \cdot \boldsymbol{p}$ model in order to acquire 
knowledge on the 2D confined levels in the QW and to position them properly with respect to $3 \mathrm{D}$-like band edges in the surrounding layers. Those are confronted with the results from photoreflectance and photoluminescence experiments.

\section{Investigated structures and experimental details}

The investigated structure was grown on a (100)oriented InAs substrate in a solid source molecular beam epitaxy system equipped with valved cracker cells for both antimony and arsenic. The structure contains five type-II QWs designed in a common "W-shaped" scheme [1], separated from each other by a $25 \mathrm{~nm}$ thick $\mathrm{GaAs}_{0.08} \mathrm{Sb}_{0.92}$ layer lattice-matched to InAs. Each quantum well consists of two $1.3 \mathrm{~nm}$ wide InAs layers that confine electrons and one $3.5 \mathrm{~nm}$ wide quaternary layer of $\mathrm{Ga}_{0.80} \mathrm{In}_{0.20} \mathrm{As}_{0.15} \mathrm{Sb}_{0.85}$ for confinement of holes. These layers are sandwiched between $2.5 \mathrm{~nm}$ thick AlSb barriers. The layer sequence of a singe well is shown schematically in Fig. 1.

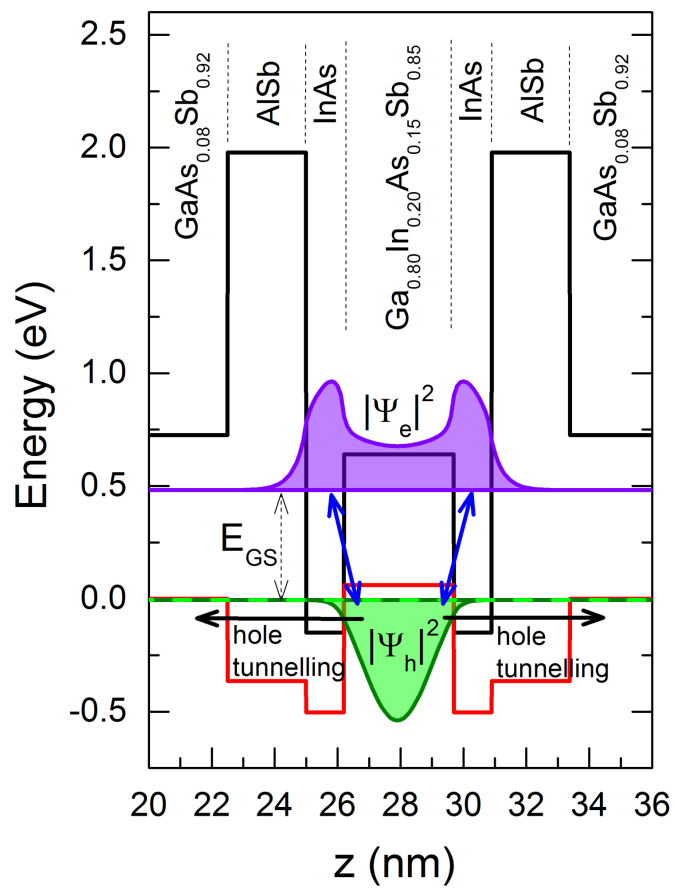

Fig. 1. Calculated band structure of a single W-type $\mathrm{AlSb} / \mathrm{InAs} / \mathrm{Ga}_{0.80} \mathrm{In}_{0.20} \mathrm{As}_{0.15} \mathrm{Sb}_{0.85} / \mathrm{InAs} / \mathrm{AlSb}$ quantum well for room temperature parameters. $\left|\Psi_{\mathrm{e}}\right|^{2}$, and $\left|\Psi_{\mathrm{h}}\right|^{2}$ are probability functions for an electron (violet area) and a hole (green area) on their fundamental levels confined in the well. Blue vertical double arrows indicate possible optical transition at the quantum well ground state. Hole tunneling towards GaAsSb is indicated by black horizontal arrows.

Calculation of the QW band structure has been performed within the 8 -band $\boldsymbol{k} \cdot \mathbf{p}$ model including strain [7] by using the finite difference method for solving the Schrödinger equation [8].
The photoreflectance $(\Delta R / R)$ spectrum near the fundamental edge of the $\mathrm{QW}$ is measured in the socalled bright configuration of the experimental setup. The structure was illuminated with a $250 \mathrm{~W}$ halogen lamp and additionally excited by a $650 \mathrm{~nm}$ continuouswave laser line modulated at $\approx 1 \mathrm{kHz}$. The light reflected off the sample was dispersed by a single-grating $0.55 \mathrm{~m}$ focal length monochromator and detected by a liquid nitrogen-cooled InSb single channel photodetector $(\mathrm{PhD})$, utilizing the lock-in technique. The same setup has been employed in order to measure the photoluminescence (PL) spectrum of the quantum well.

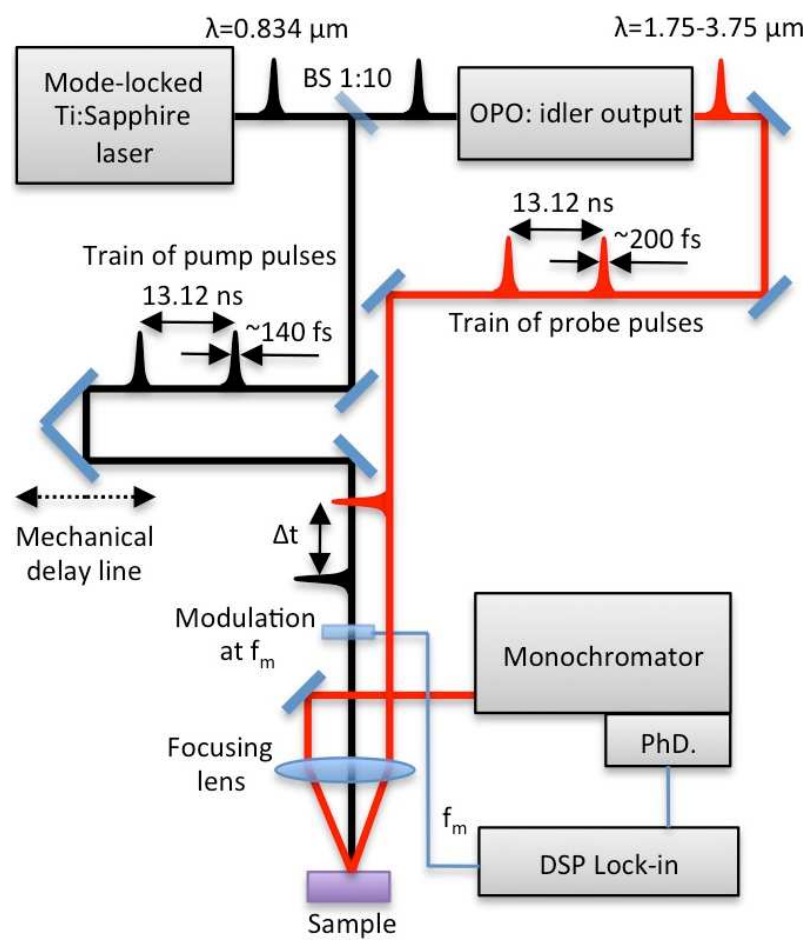

Fig. 2. Scheme of the non-degenerated pump-probe experimental setup employed for the measurement of carrier kinetics in the mid-infrared spectral range.

The non-degenerated pump-probe spectroscopic technique in the mid-infrared spectral range has been employed in order to trace carrier kinetics in the QW at room temperature. The experimental setup is schematically presented in Fig. 2. The output beam of the modelocked fs laser (Coherent Mira-HP) is split into two beams with the power ratio of $1 / 10$. The low power pump pulse train is characterized by $\mathrm{a} \approx 140$ fs pulse duration, a $13.12 \mathrm{~ns}$ pulse-to-pulse distance, and a $0.834 \mu \mathrm{m}$ photon wavelength $(\approx 1.487 \mathrm{eV})$. The high power pulse train synchronously seeds the commercially available optical parametric oscillator (OPO) (APE/Coherent OPO-HP) operating in the idler mode in order to generate a probe pulse train consisting of $\approx 200$ fs-long pulses in the midinfrared spectral range. The OPO has the capability to tune and stabilize the output photon wavelength in the range of $\approx 1.75-3.75 \mu \mathrm{m}(0.7-0.33 \mathrm{eV})$. The pump and 
probe pulses are linearly polarized and focused by a single lens on the sample surface to a spot size of $\approx 150 \mu \mathrm{m}$ in diameter. The time delay $(\Delta t)$ between coincidences of pump and probe pulses in the sample's volume is controlled by a precise $30 \mathrm{~cm}$ long mechanical delay line stage that provides $\Delta t$ in the range of $\approx 13$ fs to $\approx 2 \mathrm{~ns}$. It is realized by varying the length of the pump optical path with respect to the probe one. The time resolution of the entire setup is restricted to the probe pulse width of $\approx 200 \mathrm{fs}$ in this case, excluding accuracy related to a data processing method. Information concerning carrier kinetics in the investigated structure after its excitation by a pump pulse is provided by the analysis of the intensity of the reflected probe pulse as a function of $\Delta t$. First, the probe pulse is spectrally filtered by a $0.55 \mathrm{~m}$ focal length monochromator operating in the near-infrared spectral range, and then the respective intensity is measured by a liquid nitrogen-cooled InSb photodiode utilizing a lock-in amplifier operating at a frequency of $1 \mathrm{kHz}$, provided by a mechanical chopper mounted in the pump beam path. The lock-in technique provides a sufficiently high signal-to-noise ratio and allows to measure changes in the reflected signal on the level of $\Delta R / R \approx 10^{-6}$, as required by the experimental conditions. Note that $\Delta R / R$ is $\approx 10^{-4}$ for the investigated sample, as presented in Fig. 3.

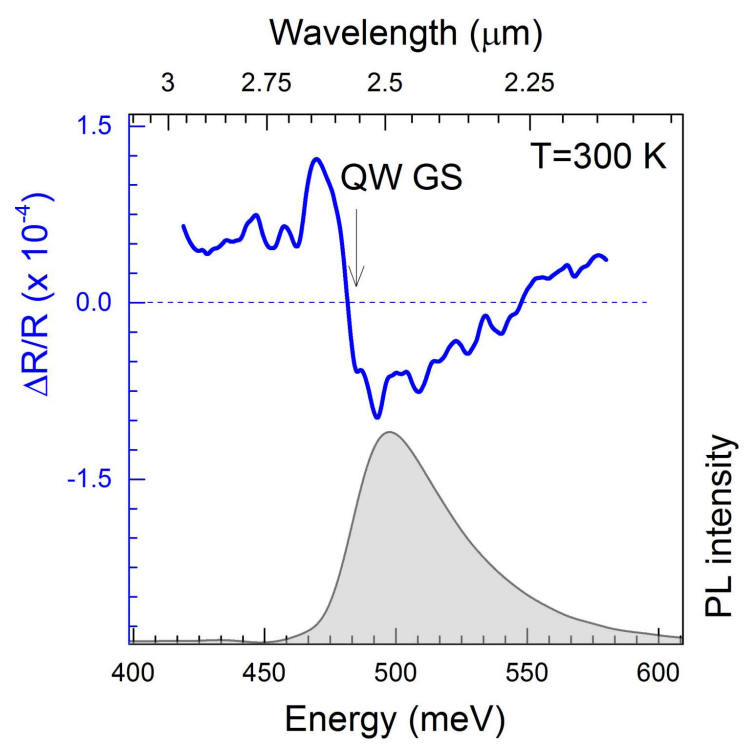

Fig. 3. Room temperature photoreflectance $(\Delta R / R)$ and photoluminescence $(\mathrm{PL})$ spectra in the vicinity of the AlSb/InAs $/ \mathrm{Ga}_{0.80} \mathrm{In}_{0.20} \mathrm{As}_{0.15} \mathrm{Sb}_{0.85} / \mathrm{InAs} / \mathrm{AlSb}$ quantum well ground state.

\section{Experimental results and discussion}

A theoretical evaluation of the band structure for the investigated QW system restricted to a single well is shown in Fig. 1. The band alignment mimics the "W" letter that typically names the whole family of similar quantum systems. The fundamental optical transition in this QW appears at $0.482 \mathrm{eV}$ at room temperature.
One can see that the highest hole state is almost entirely located in the $\mathrm{Ga}_{0.80} \mathrm{In}_{0.20} \mathrm{As}_{0.15} \mathrm{Sb}_{0.85}$ layer, as indicated by the probability density function $-\left|\Psi_{h}\right|^{2}$ (green area in Fig. 1). The lowest electron state is smeared over the $\mathrm{Ga}_{0.80} \mathrm{In}_{0.20} \mathrm{As}_{0.15} \mathrm{Sb}_{0.85}$ material, with the peaks of the probability density - $\left|\Psi_{e}\right|^{2}$ located in both InAs layers (violet area in Fig. 1). Thus, the expected ground state transition between the $2 \mathrm{D}$ electron and hole distributions in the QW, indicated by vertical double arrows in Fig. 1, is in fact indirect in real space. One must note that the position of the top of the valence band in the $\mathrm{GaAs}_{0.08} \mathrm{Sb}_{0.92}$ barrier is close in energy to the fundamental 2D hole state confined in the well and the spatial distance between $\mathrm{Ga}_{0.80} \operatorname{In}_{0.20} \mathrm{As}_{0.15} \mathrm{Sb}_{0.85}$ and the barrier is only $3.8 \mathrm{~nm}$, thus one may expect electronic coupling between both types of states. This is very unlikely in the case of electrons, where the existence of some middle-gap states in $\mathrm{GaAs}_{0.08} \mathrm{Sb}_{0.92}$ is required for a similar effect to be present, since the bottom of the conduction band in $\mathrm{GaAs}_{0.08} \mathrm{Sb}_{0.92}$ is about $250 \mathrm{meV}$ higher than the fundamental 2D state for an electron confined in the well.

In order to verify the output of the theoretical analysis one can perform photoreflectance and photoluminescence experiments in the vicinity of the QW fundamental transition at room temperature. Figure 3 presents $\Delta R / R$ (blue curve) and $\mathrm{PL}$ spectra (black curve) of the investigated $\mathrm{AlSb} /$ InAs $/ \mathrm{Ga}_{0.80} \mathrm{In}_{0.20} \mathrm{As}_{0.15} \mathrm{Sb}_{0.85} / \mathrm{InAs} / \mathrm{AlSb} \mathrm{QW}$. A strong $\Delta R / R$ feature is observed at the energy of $0.485 \mathrm{eV}$ $(\approx 2.56 \mu \mathrm{m}$ wavelength $)$ that corresponds very well to the calculated energy $E_{G S}$ of the QW ground state transition. The PL emission band is centered at the same energy $E_{G S}$ and shows pronounced broadening and asymmetry towards high-energy tail related to the electronhole recombination process that occurs within certain thermal distribution of carriers.

Carrier kinetics in the AlSb/InAs/ $\mathrm{Ga}_{0.80} \mathrm{In}_{0.20} \mathrm{As}_{0.15} \mathrm{Sb}_{0.85} / \mathrm{InAs} / \mathrm{AlSb} \mathrm{QW}$ has been measured in the non-degenerated pump-probe reflectance (TRR) experiment in which changes in the population of a certain QW state are seen through the reflectivity changes of the probe pulse induced by absorption and thus refractive index nonlinearities [9]. In the experiment, the pump pulse with an energy of $P_{\text {pump }} \approx 100 \mathrm{pJ}$ and a photon energy of $1.467 \mathrm{eV}$ creates an initial free and hot carrier population at the higher QW states. A weak probe pulse $\left(P_{\text {probe }} \approx 10 \mathrm{pJ}\right)$ tuned energetically to the GS of the QW at $\approx 0.485 \mathrm{eV}$ $(\approx 2.56 \mu \mathrm{m})$ subsequently tracks the carrier population build-up at the GS and its subsequent decay due to various possible relaxation channels in time.

Figure 4 presents the measured TRR signal at $T=$ $300 \mathrm{~K}$ consisting of a rapidly rising amplitude, followed by two significantly different decay components. An initial increase in the TRR signal, characterized by the time constant of $\tau_{\text {rise }}=2.3 \pm 0.2 \mathrm{ps}$, is related to the build-up of a certain carrier population at the QW GS. Omitting the effect of carrier diffusion within the 2D 
states, such rapid initial relaxation is probably governed by phonon (optical or acoustic) scattering rather than Auger-type relaxation due to the low initial density of photoinjected carriers. A similar time constant of $\approx 1.2 \mathrm{ps}$ for the initial carrier cooling via optical phonon emission has been obtained during the study of (GaInSb/InAs)/GaInAlAsSb multiple quantum wells [3]. After obtaining the quasi-equilibrium condition among the carrier population at the QW GS, electron and holes further relax, however in a much longer time scale. The long lasting component of the TRR signal, tracked up to $1.6 \mathrm{~ns}$, is attributed to the radiative recombination of spatially separated electron-hole pairs (electrons in the InAs layer and holes in $\left.\mathrm{Ga}_{0.80} \operatorname{In}_{0.20} \mathrm{As}_{0.15} \mathrm{Sb}_{0.85}\right)$. It is expected that the radiative recombination of an electronhole pair at room temperature in these type-II quantum wells can reach an average value of a few to tens of ns $[2,4-6]$. The most intriguing and not observed before relaxation channel is indicated by the short decay component of the TRR signal. The time-constant related to this component is $240 \pm 10$ ps. One can point out at least two possible reasons leading to such an observation: (i) carrier escape from the QW to some population of e.g. optically inactive states spatially located at the InAs $/ \mathrm{Ga}_{0.80} \mathrm{In}_{0.20} \mathrm{As}_{0.15} \mathrm{Sb}_{0.85}$ heterointerface [10-12], or (ii) it can be attributed, as recently postulated [13], to the hole tunnelling out of its confining potential towards the $\mathrm{GaAs}_{0.08} \mathrm{Sb}_{0.92}$ matrix. The first scenario cannot be totally excluded because the existence of non-radiative centres at the QW interfaces has already been confirmed in several experiments $[10,12]$. However, there is lack of knowledge concerning the density of such states and their nature, which would be useful for e.g. modelling of carrier kinetics. The most intriguing is the second possibility and it is worth investigating more in detail.

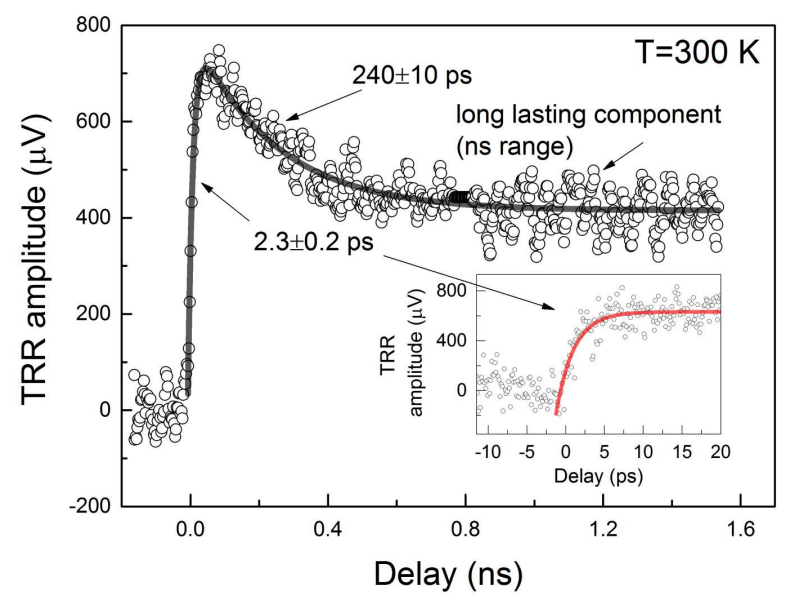

Fig. 4. Time-resolved reflectivity (TRR) trace measured at $T=300 \mathrm{~K}$ for the $\mathrm{AlSb} / \mathrm{InAs} / \mathrm{Ga}_{0.80} \mathrm{In}_{0.20} \mathrm{As}_{0.15} \mathrm{Sb}_{0.85} / \mathrm{InAs} / \mathrm{AlSb}$ quantum well. The photon wavelength of pump and probe pulses are $0.834 \mu \mathrm{m}$ and $2.56 \mu \mathrm{m}$, respectively. $P_{\text {pump }}=100 \mathrm{pJ}, P_{\text {probe }}=10 \mathrm{pJ}$.
Figure 1 indicates the possibility of a hole tunnelling out of its $2 \mathrm{D}$ confined potential in the well towards the $3 \mathrm{D}$ density of states near the top of the $\mathrm{GaAs}_{0.08} \mathrm{Sb}_{0.92}$ valence band (black arrows). One can check this hypothesis by estimating the hole tunnelling/escape time within the framework of the Wentzel-Kramers-Brillouin approximation and compare the result with the experimental data. In this case the tunnelling barrier is defined by two $d_{\text {InAs }}=1.3 \mathrm{~nm}$ wide InAs and $d_{\mathrm{AlSb}}=2.5 \mathrm{~nm}$ wide AlSb layers. Therefore, the hole transmission coefficient through the barrier is calculated according to the following expression [14]:

$$
T=\frac{16 E\left(V_{\mathrm{b}}-E\right)}{V_{\mathrm{b}}^{2}} \exp \left(-2 d_{\mathrm{b}} \sqrt{2 m_{\mathrm{h}}\left(V_{\mathrm{b}}-E\right)} / \hbar\right),
$$

where $E$ is the energy distance from the bottom of the valence band of the $\mathrm{Ga}_{0.80} \mathrm{In}_{0.20} \mathrm{As}_{0.15} \mathrm{Sb}_{0.85}$ material to the first confined state for holes in the well, $V_{\mathrm{b}}$ is the effective confinement depth for a hole, $d_{\mathrm{b}}$ is the barrier width: $d_{\mathrm{b}}=d_{\mathrm{InAs}}+d_{\mathrm{AlSb}}$, and $m_{\mathrm{h}}$ is the hole effective mass in the barrier. For a given set of parameters: $E=57 \mathrm{meV}, V_{\mathrm{b}}=432 \mathrm{meV}$, and $m_{\mathrm{h}}=0.2 m_{0}$, the calculated transmission coefficient equals $T=5.5 \times 10^{-5}$ which can be translated into the tunnelling/escape time [14]:

$$
\tau_{\mathrm{TUN}}=\left(\frac{2 d_{\mathrm{b}}}{T \sqrt{2 E m_{\mathrm{h}}^{-1}}}\right) .
$$

Thus, the calculated hole tunneling time results to $400 \mathrm{ps}$, which is reasonably close to $240 \pm 10 \mathrm{ps}$, as measured for the fast decay component of the TRR signal, and therefore can support the relaxation scenario through the out-tunneling of holes from their 2D confinement potential. The apparent discrepancy between these numbers can arise from the employed theoretical approximation that depends on several different parameters, most importantly the effective hole mass. Moreover, the experimentally obtained time-constant can be also influenced by some additional fast relaxation channels, like the already discussed carrier escape towards defect-like states at the $\mathrm{InAs} / \mathrm{Ga}_{0.80} \mathrm{In}_{0.20} \mathrm{As}_{0.15} \mathrm{Sb}_{0.85}$ heterointerface.

\section{Conclusions}

Several experimental and theoretical approaches have been used to study the band structure and carrier dynamics at room temperature in the W-type $\mathrm{AlSb} / \mathrm{InAs} / \mathrm{GaInAsSb} / \mathrm{InAs} / \mathrm{AlSb}$ quantum well emitting in the mid-infrared spectral range. The results of the non-degenerate pump-probe experiment in the reflectance configuration show a fast $2.3 \pm 0.2$ ps carrier relaxation time from the upper to the ground state of the well, driven, most probably, by the carrier-phonon (optical or acoustic) scattering processes. Two relaxation channels seem to be responsible for the decay of a quasiequilibrium carrier population at the QW ground state. 
The first one is related to the radiative recombination of the spatially separated electron-hole pairs occurring in the ns time scale. The more interesting and unusual, however, is the second relaxation channel, characterized by a $230 \pm 10$ ps decay time constant, which is attributed to the tunneling of a hole out of its $2 \mathrm{D}$ confining potential towards the distant $\mathrm{GaAs}_{0.08} \mathrm{Sb}_{0.92}$ barrier, which has not been observed in such a family of $\mathrm{W}$-type quantum wells.

\section{Acknowledgments}

This project has received funding from the European Commission's Horizon 2020 Research and Innovation Programme iCspec under grant agreement No. 636930 and has also been supported by the National Science Centre of Poland within Grant No. 2014/15/B/ST7/04663.

\section{References}

[1] I. Vurgaftman, W.W. Bewley, C.L. Canedy, C.S. Kim, C.D. Merritt, J. Abell, J.R. Lindle, J.R. Mayer $\mathrm{Na}$ ture Commun. 2, 585 (2011).

[2] S.W. McCahon, S.A. Anson, D.-J. Jang, M.E. Flatté, T.F. Boggess, D.H. Chow, T.C. Hasenberg, C.H. Grein, Appl. Phys. Lett. 68, 2135 (1996).

[3] D.-J. Jang, J.T. Olesberg, M.E. Flatté, T.F. Boggess, T.C. Hasenberg, Appl. Phys. Lett. 70, 1125 (1997).

[4] J.R. Meyer, C.L. Felix, W.W. Bewley, I. Vurgaftman, E.H. Aifer, L.J. Olafsen, J.R. Lindle, C.A. Hoffman, M.-J. Yang, B.R. Bennett, B.V. Shanabrook, H. Lee, C.-H. Lin, S.S. Pei, R.H. Miles, Appl. Phys. Lett. 73, 2857 (1998).
[5] M.E. Flatté, C.H. Grein, T.C. Hasenberg, S.A. Anson, D.-J. Jang, J.T. Olesberg, T.F. Boggess, Phys. Rev. B 59, 5745 (1999).

[6] W.T. Cooley, R.L. Hengehold, Y.K. Yeo, G.W. Turner, J.P. Loehr, Appl. Phys. Lett. 73, 2890 (1998).

[7] G.L. Bir, G.E. Pikus, Symmetry and Strain-Induced Effects in Semiconductors, Wiley, New York 1974.

[8] S.L. Chuang, C.S. Chang, Semicond. Sci. Technol. 12, 252 (1997).

[9] B.R. Bennett, R.A. Soref, J.A. del Alamo, IEEE J. Quantum Electron. 26, 113 (1990).

[10] W. Barvosa-Carter, M.E. Twigg, M.J. Yang, L.J. Whitman, Phys. Rev. B 63, 245311 (2001).

[11] M. Motyka, G. Sęk, K. Ryczko, M. Dyksik, R. Weih, G. Patriarche, J. Misiewicz, M. Kamp, S. Hofling, Nanoscale Res. Lett. 10, 471 (2010).

[12] X. Junliang, Z. Yu, L. Yongping, W. Juan, X. Wei, H. Hongyue, X. Yingqiang, N. Zhichuan, J. Appl. Phys. 116, 123107 (2014).

[13] G. Sęk, F. Janiak, M. Motyka, K. Ryczko, J. Misiewicz, A. Bauer, S. Höfling, A. Forchel, Opt. Mater. 33, 1817 (2011).

[14] J. Shah, Ultrafast Spectroscopy of Semiconductor and Semiconductor Nanostructures, Springer, New Jersey 1996. 